\title{
Signet Ring Cell Carcinoma Arising from a Solitary Juvenile Polyp in the Colon
}

\author{
Hae Jung Kim, Min Kwan Kang, Hee Suk Lee, Do Sun Kim, Du Han Lee \\ Daehang Clinic, Seoul, Korea
}

Juvenile polyps are relatively common polyps that affect predominantly young patients and may occur in isolated, multiple, and/or familial forms. They have been considered to be benign lesions without neoplastic potential, but for patients with multiple juvenile polyposis, the cumulative malignant risk is greater than fifty percents. In patients with a solitary polyp, the risks are minimal, and only a few cases of malignant change from a solitary juvenile polyp have been reported. We describe the case of a twenty one year old female with one solitary juvenile polyp, which contained a signet ring cell carcinoma in the mucosal layer.

Keywords: Colonic polyps; Solitary juvenile polyps; Carcinoma; Signet ring cell

\section{INTRODUCTION}

After the first report by Diamond [1] in 1939, juvenile polyps, one of the polyps found in the large intestine, have been reported to occur in relatively young people. Juvenile polyps may appear as solitary lesions, multiple polyps or familiar polyposis. The juvenile polyp is a type of harmatoma that develops in the connective tissue of the large intestinal submucosal layer, but its etiology is not clear. In a recent study, multiple juvenile polyposis was reported to have a cumulative malignant potential in $50 \%$ of the patients. On the other hand, a solitary lesion is thought to a benign disease with low malignant potential, and it can be treated sufficiently by using only an endoscopic polypectomy, with no follow-up observation being required. In a patient admitted for the chief complaint of anal bleeding, we detected polyps in the sigmoid colon and performed an endoscopic polypectomy. By histopathologic test, the polyp was diagnosed as a harmatoma with a focal signet ring cell

Received: March 1, 2010 Accepted: July 31, 2010

Correspondence to: Hae Jung Kim, M.D.

Daehang Clinic, 481-10 Bangbae 3-dong, Seocho-gu, Seoul 137-820, Korea

Tel: +82-2-6388-8565, Fax: +82-2-6388-8673

E-mail: hjkim@daehang.com

(C) 2010 The Korean Society of Coloproctology

This is an open-access article distributed under the terms of the Creative Commons Attribution NonCommercial License (http://creativecommons.org/licenses/by-nc/3.0) which permits unrestricted non-

commercial use, distribution, and reproduction in any medium, provided the original work is properly cited. carcinoma limited to the mucosal layer. This case is reported as a rare case of malignant degeneration of a solitary juvenile polyp.

\section{CASE REPORT}

A 21-year-old female visited our hospital with a chief complaint of bleeding on defecation. In digital rectal examination, no hemorrhoid, anal fissure or other anal disease was found, but blood-tinged stool was detected. For several days, she had had symptoms of diarrhea, with no abdominal pain or febrile sense. She had no past medical history of polyps or abdominal surgery. None of her family had had inflammatory bowel diseases, multiple polyposis, malignant diseases in the digestive tract, except that her mother had undergone breast cancer surgery. On colonoscopic examination, a pedunculated polyp was detected in the sigmoid colon. The polyp had two branching heads, $20 \mathrm{~mm}$ and $27 \mathrm{~mm}$ in size respectively, and the surface was relatively smooth, but covered with mucous discharge. In addition, a lobulating change, different from that in typical juvenile polyps, was found (Fig. 1).

After a detached snare had been applied, a snare polypectomy was done in the neck of the polyp. During the polypectomy, no hemorrhage or abdominal pain developed, and the patient was discharged one day after the procedure. On pathologic examination, the polyp was diagnosed as a juvenile polyp that had several branches, multiple cystic changes, and interstitial 


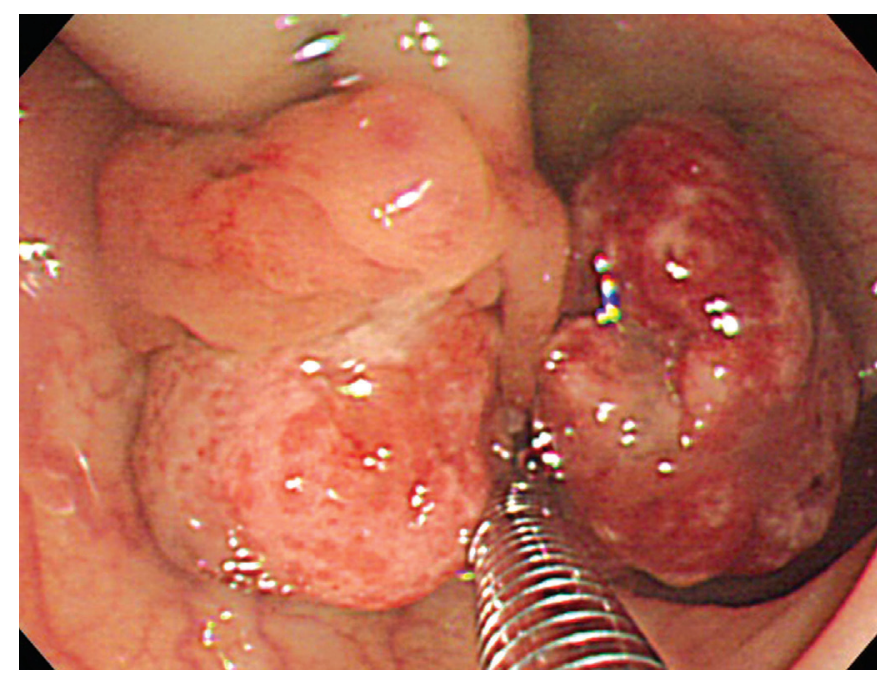

Fig. 1. Pedunculated polyp with long stalk was found in sigmoid colon. Two round lobulating heads covered with whitish patches are noted.

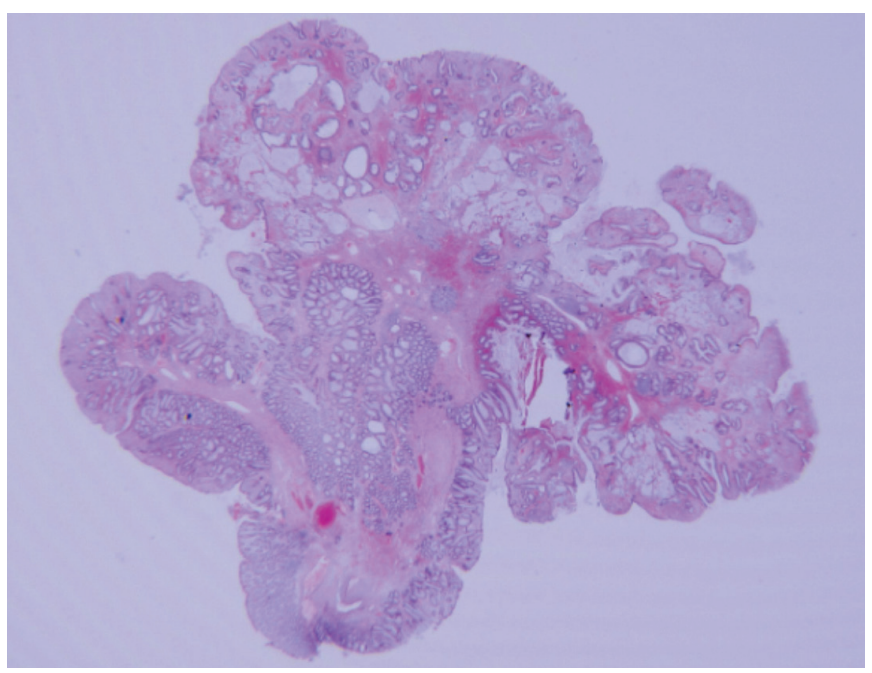

Fig. 2. Polyp showing multilobulation with normal elements of the colonic mucosa and ecstatic glands filled with mucin $(\mathrm{H} \& \mathrm{E}, \times 1)$.

edema with mucinous congestion and infiltration of acute and chronic inflammatory cells (Fig. 2). In some areas of the polyp, local infiltration of a signet ring cell carcinoma into the mucosa was observed, but there was no adenomatous change (Fig. 3). On abdominal CT performed after the polypectomy, no distant metastasis or infiltration to adjacent tissues was detected, and serum level of carcinoembryonic antigen or CA19-9 was not elevated. In the follow-up endoscopic examination performed 3 months later, no recurrence at the polypectomy site or residual polyps were found; the patient is still under outpatient follow-up observation.

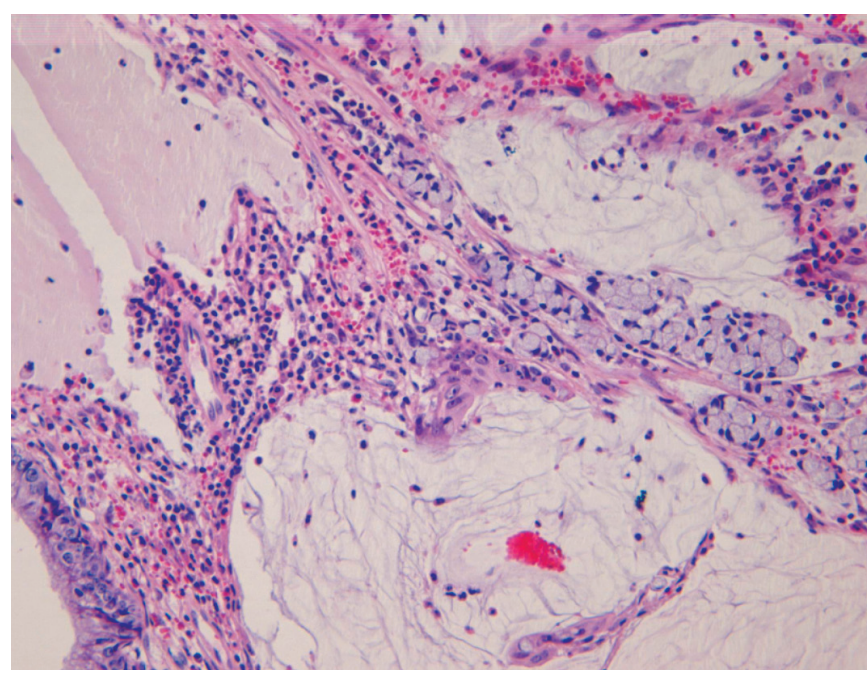

Fig. 3. Laminar propria showing numerous mucinous pools with floating and infiltrating signet ring cells. Some inflammatory cells are also infiltrated $(\mathrm{H} \& \mathrm{E}, \times 200)$.

\section{DISCUSSION}

Horrilleno et al. [2] termed pediatric polyps showing the formation of cysts by the epithelial grands with abundant mucoid, increased connective tissues, and chronic infiltration of eosinophils as juvenile polyposis, and Morson [3] referred to such polyps showing hyperproliferation of normal tissues in the upper part of interstitial tissue as a harmatoma. Afterward, McColl et al. [4] reported multiple juvenile polyposis, which was considered to be a different disease from multiple adenomatosis. Still later, Sachatello [5] reported a familial juvenile polyposis.

The most frequent symptom of juvenile polyposis is hemorrhage in the rectum, as shown in our case; anal prolapse, abdominal pain, diarrhea, mucous stool, rectal prolapse, and other symptoms may also develop. In approximately $10 \%$ of the patients, spontaneous amputation was reported. Juvenile polyposis in the large intestine is detected in the distal rectum in most cases. Typical juvenile polyps have a round or an oval pedunculated form with a smooth continuous surface, and numerous microcysts filled with mucoid are detected in a cross section. In addition, in $20 \%$ of the cases, multicentric segments or branches are observed. In microscopic findings in the upper part of the mucularis mucosa, glandular epithelial ducts with columnar epithelial cells and goblet cells are found, and they show cystic dilatation and are filled with mucin and numorous inflammatory cells. These are benign lesions if cellular hyperproliferation, hypercchromatism, and excessive cell division of the epithelial cells covering the surface are not present, and they can be sufficiently treated by using only a colonoscopic polypectomy. Since the malignant deterioration potential is very low, follow-up observation is not required. 
Approximately $30 \%$ of juvenile polyposis cases have multiple polyps at the time of diagnosis. When such juvenile polyposis occurs in a familial form, it is inherited as an autosomal dominant trait. Approximately $25-50 \%$ of the cases are associated with adenoma or dysplasia with a malignant potential; thus, they are considered as precancerous lesions. In addition, in multiple polyposis, besides the large intestine, polyps may develop in the entire digestive system in some cases; thus, if a family history is suspected or genetic mutation is confirmed, for the assessment of multiple lesions, an overall examination of the esophagus, stomach, small intestine and large intestine is required.

The cause of the development of cancer in juvenile polyposis has not been elucidated yet. The surface ulcer observed in some polyps, infection and necrosis are thought to be causatives inducing an inflammatory reaction or infiltration. According to Jass et al. [6], the malignant potential of a solitary juvenile polyp is very low. Nonetheless, Jones et al. [7] reported adenomatous changes and cases of signet ring cell carcinoma limited to the mucosal layer, but in our case, adenomatous changes were not found. The mechanism of malignant deterioration or carcinomatous changes in solitary polyps is thought not to be different from the mechanism in juvenile polyposis. Although it is very rare, the possibility of malignant deterioration of solitary juvenile polyposis should be kept in mind, and in a polypec- tomy, attention should be paid to securing sufficient resection margins. In addition, comprehensive histological examination and follow-up observation are required.

\section{CONFLICT OF INTEREST}

No potential conflict of interest relevant to this article was reported.

\section{REFERENCES}

1. Diamond M. Adenoma of the rectum in children: report of a case in a thirty month old girl. Am J Dis Child 1939;57:360-7.

2. Horrilleno EG, Eckert C, Ackerman LV. Polyps of the rectum and colon in children. Cancer 1957;10:1210-20.

3. Morson BC. Some peculiarities in the histology of intestinal polyps. Dis Colon Rectum 1962;5:337-44.

4. McColl I, Busxey HJ, Veale AM, Morson BC. Juvenile polyposis coli. Proc R Soc Med 1964;57:896-7.

5. Sachatello CR. Polypoid diseases of the gastrointestinal tract. J Ky Med Assoc 1972;70:540-4.

6. Jass JR, Williams CB, Bussey HJ, Morson BC. Juvenile polyposis: a precancerous condition. Histopathology 1988;13:619-30.

7. Jones MA, Hebert JC, Trainer TD. Juvenile polyp with intramucosal carcinoma. Arch Pathol Lab Med 1987;111:200-1. 